\title{
The relationship between Probability and Mathematical Statistics and Professional Courses in Economic Management
}

\author{
QI ZHAO \\ Department of Foundation, Shandong Yingcai University, Jinan 250104, China.
}

Key words: Probability theory and mathematical statistics, Economy and Management Specialty course, Reform in Education

\begin{abstract}
In this paper, we study how Probability theory and mathematical statistics courses combined with the professional course in application oriented university of economic management specialty. According to the relationship between probability and statistics courses and the professional course, The economic and management courses are classified, on this basis ,Put forward suggestions for the reform of probability theory and mathematical statistics teaching contents, teaching methods and assessment methods.
\end{abstract}

Probability and mathematical statistics is a professional basic course of Economic management professional, Concepts and methods of the probability theory and mathematical statistics were used many course of Economic management professional, So to learn probability theory and mathematical statistics play a very important role for specialized course learning. In the face of applied undergraduate college students, As a teacher, Should consider the different requirements of different professional, Teaching content can't completely the same. Applied undergraduate colleges and universities aims to "apply", Teachers in the teaching process should fully reflect this, At first, should know what courses involves to probability and mathematical statistics, And then to carry out targeted teaching

1 The relationship between Probability and mathematical statistics and Economic management professional courses

At present, the economy of our country most universities and colleges of undergraduate course specialized courses in professional involves to probability have more than 20 courses, The twenty more courses can be roughly divided into four categories.

1.1 The first type of courses Involves the concept of probability theory and thinking methods but Involves less quantitative calculation

Risk is a common concept in professional course of Economic management professional, Such as risk management, risk decision-making and so on. The concept of risk has a slightly different expression in different courses, but risk comes from the uncertainty on the whole. Probability and mathematical statistics is the study of uncertainty phenomenon statistical regularity, So learn probability theory and mathematical statistics of the relevant concepts of random phenomenon help to understand the course of these concepts. Involved in courses and related content is as follows:

(1) Entrepreneurship management: Entrepreneurship operational risk management

(2) International financial management: foreign exchange risk and type, foreign exchange risk management strategy

(3) Microeconomics: the uncertainty and risk analysis

(4) Foundation of Assets evaluation: long-term bond risk assessment

(5) Principles of management: Risk type decision method: there are two main methods, decisionmaking method of income statement and the decision tree method

(6) Introduction to e-commerce: The risk of e-commerce

(7) Fiscal and financial: security is one of the three big principles in commercial Banks, Security is 
to point to a bank assets the degree avoid the risk

(8) Project management: The concept of risk in project risk management

(9) enterprise culture: Enterprise risk and risk management

(10) Science of auditing: Audit risk is financial statements is mistake but Certified public accountants issued the possibility of improper audit opinions. The risk of audit sampling: In the control test ,Audit sampling have two main risk, one is overreliance, another is lack of trust. In the substantive tests, Audit sampling also have two main risk, one is Wrong refused to, another is Wrong to accept

1.2 The second type of courses Involves the concept of Probability and mathematical statistics and all kinds of quantitative calculation, Mainly risk measures, expected earnings

Variance or standard deviation reflects the random variables deviate from the expectation average deviation degree, People usually use variance or standard deviation to measure risk. Mathematical expectation reflects the average of a random variable values, Many quantity of economics is a random variable, for example, earnings, The average earnings is mathematical expectation of the earnings. Involved in courses and related content is as follows:

(1) security analysis and investment: mean value - the standard deviation model of portfolio theory, Harry M. Markowitz use expected yield and standard deviation, respectively to measure the returns and risk of investment portfolio

(2) Modern logistics: Risk type decision of logistics decision, risk type decision refers to the decision environment is not entirely sure, but the probability of occurrence is known. The maximum expected return criterion in risk decision. The first, list the corresponding earnings decision matrix of "strategy $\longrightarrow$ event", i.e. The income statement. The second, According to the statistical analysis of historical data to determine the probability of different events. The third, Calculation of expected profit of each scheme, Calculating formula for $\sum_{j} p_{j} a_{i j}, i=1,2, \ldots, n$. The fourth, Select the expected profit maximum, the value of the corresponding strategies for the optimal strategy, i.e.

$$
\max _{i} \sum_{j} p_{j} a_{i j} \rightarrow S_{k}^{*}
$$

(3) Managerial economics: Enterprise risk decision, risk assessment procedures and methods. To evaluate the risk, In fact is, Using the theory of probability and mathematical statistics method, Calculation and analysis of all the possible results.

(4) Tutorial financial management practice: In the financial management practice, Work in order to simplify the financial analysis and decision, Short-term financial decisions generally does not consider risk factors, But long-term financial decision-making risk factors should be considered, and determination of degree of risk. Risk is directly related to probability.

(5) Management of operational research: The storage model of random storage model, In the storage model, demand, supply, pay interval if have any random variables, it is called a random storage model.

In the queuing theory application probability and mathematical statistics knowledge

(6) Risk management: The theoretical basis of risk measurement, The law of large number and probability reasoning principle. The main numerical of quantitative risk, Variance, standard deviation and coefficient of variation. Commonly used risk value measurement method, the mean - variance method. Enterprise risk loss of static measurement method, Sample estimation and The total loss distribution method.

(7) Quality management: Quality management is commonly used in statistical method, for example, Histogram, control diagram, sampling inspection, the orthogonal test, regression analysis, the least squares method, and so on.

1.3 The third type of courses Involves sampling statistics method 
In the mathematical statistics, through the extraction part of the overall (i.e. sample), according to the sample information to make inference is a basic method. In the Economy and Management Specialty course has the following courses involve sampling statistics method.

(1) network marketing: Network dynamic pricing risk and countermeasures; network marketing research method of random IP method, it belongs to the initiative investigation method, its theory base is on random sampling.

(2) Public relations: Public relations research method of the sampling research method.

(3) fundamentals of accounting: Sampling inventory method is one of the property inventory method, The sampling method of inventory refers to take a small amount of samples from the overall, by measuring the sample size, then deduce overall method according to the sample. This method is suitable for large number, weight uniform, inconvenience one by one counting of the inventory of the property, The most common sampling inventory method is random sampling method. Random sampling method is in the overall extraction part units in inventory, On the basis of the results of the inventory one way to determine the overall indicators.

(4) Quality management: Sampling inspection of product inspection, there are two kinds of sampling risk, producers risk and consumers risk. i.e. refuse to truth and receive false.

(5) Science of auditing: Audit sampling is an important part of the science of auditing, Statistical sampling is an important part in the audit sampling method

6) Market research and prediction: sampling statistical method in the scheme design of market research, When the market sampling investigation, due to the investigation object is just a part of the overall, in part to infer the overall, there must be error, To a certain degree of reliability assurance sampling error is less than the given range, Given the range of sampling error is called the sampling error is allowed .(This part of the corresponding to the interval estimation of probability theory and mathematical statistics)

1.4 The fourth type of courses Involves prediction method of the probability theory

Regression analysis is a method of study correlativity, it is one of the most commonly used method of mathematical statistics, has been widely used. One of the application of regression analysis is to forecast and control, Most used in economic management professional course is linear regression model of a variable prediction method,( Of course there are other prediction methods).

Basically has the following course involves such content:

(1) Market research and prediction: In market research, Often want to confirm whether have correlativity between two or more variables, if there have, Researchers also want to know how much the strength of the relevant, Correlation analysis can be used to measure the intensity of the relationship between the variable. Regression analysis is a method to establish relationships between two or more than two variables, In the independent variable on the basis of the description, prediction and control the dependent variable

In addition, Market research of prediction method also have subjective probability method, and Markov prediction method.

(2) International marketing: There are a lot of data analysis methods in the international marketing information data analyze, Linear regression analysis is one of them.

In addition, There are a lot of field use monadic linear regression prediction method, for example, the logistics demand forecasting of the Modern logistics, manage prediction quantitative analysis of management accounting, Inventory demand forecasting of inventory management, Market demand estimate of the managerial economics, market forecast of marketing, and so on. Purchasing and supply management of procurement demand forecasting subjective probability method, the crossover probability method is used 


\section{Probability and mathematical statistics teaching reform}

2.1 The reform of the teaching content

Traditional mathematics teaching too much emphasis on theoretical rigor, Teachers spend a lot of time used in the interpretation of the definition, Proof of theorem and exercise calculus, Only pay attention to imparting knowledge, Often ignore the pass of mathematical thinking, Especially the application of knowledge. It is inadvisable for student of non-mathematics major, Doing so will make students feel boring, And then lost interest in learning. In fact, Probability and mathematical statistics is a discipline own very strong practical, closely connected with practical subject is one of its development power. In the teaching practice, Teachers should make students understand the application of Probability and mathematical statistics in the professional course, Improve students' using probability theory and mathematical statistics knowledge ability to solve actual problems, only in this way, to comply with the goals of training high level applied talents.

On the teaching content should increase the contents of the statistical, Statistics closely related to economic management specialty. Now most schools in probability and mathematical statistics on the scheduling is unreasonable, the probability taken up too much class hour, the statistical part of class time is not enough. Should be appropriate to reduce the content of the probability theory part, Delete the classical probability of complex calculation, Delete distribution function and probability density function of complex calculation. For theory knowledge, Should explain to students from the perspective of intuitive and easy to understand.

2.2 The reform of the teaching methods and teaching means

With the development of computer technology, Multimedia aided teaching has gradually become the mark of modern teaching. Although in the mathematics teaching of how to develop multimedia auxiliary teaching has been some debate, Today's college mathematics classroom is dominated by multimedia courseware is an indisputable fact. There are many phenomena in probability and mathematical statistics can through the computer simulation, to do so is helpful for students to understand the regularity of knowledge. For example, Buffon throw needle experiment shows that frequency stability, Using Poisson distribution to approximate binomial distribution, etc. Use the multimedia auxiliary teaching, to show some random phenomena in the teaching process, be able to put some questions of difficult to explain more intuitively, Both save precious class time, and keep the knowledge systematic, coherence and fluency, To arouse the curiosity of students, improve the students' understanding of knowledge.

More case teaching in teaching methods, Make the teaching content connection professional courses, in view of the economic management specialty students, choosing proper case in the classroom teaching. The teacher should be to search related case from the students' specialized courses.

2.3 Assessment of the reform of the way

Teaching reform is difficult to promote, a very important reason is that the inspection way lagging. Exam-oriented education also shrouded in the today's university, the students still continue "endorsement - test - forget" routines. So, Probability and mathematical statistics examination way also to reform, not only have closed-book examination, also should have open-book examination And other examination way. For example, the teacher can set some practical problems, let the students have written essay, and so on. In a word, As long as is helpful to arouse the enthusiasm and initiative of student learning, Is conducive to the cultivation of the students application ability, evaluation mode may flexible and varied.

*Project supported by the statistical scientific research project of Shandong province(project No.KT16138)

\section{References}


[1] G. CH. Wu, Probability and mathematical statistics. Renmin university of China press, Beijing, 2011

[2] K. H. Zeng, Fiscal and financial. China financial and economic publishing house, Beijing,2009

[3] Y. Wang, security analysis and investment. Beijing university press, Beijing, 2012

[4] Z. P. Hao, X. L. Liu, Science of auditing. Beijing university press, Beijing, 2013

[5] Y. Chen, Marketing. Beijing university press, Beijing, 2012

[6] F. Xie, Theory and method of Risk management. Chongqing university press, Chongqing, 2013

[7] M. Cheng, Project management, Beijing university press, Beijing, 2013

[8] W. Wei, Microeconomics, Wuhan university press, Wuhan, 2011

[9] Y. M. Zhou, X. M. Hu, Assets evaluation, Shanghai university of finance and economics press, 2010

[10] J. Wu, Modern logistics, Beijing university press, Beijing, 2010

[11] X. J. Cao, Market research and prediction, The communication university of China press, Beijing, 2010

[12] Q. ZH. Liang, W. H. ZH, Entrepreneurship management, Beijing university press, Beijing, 2007

[13] Q. Li, L. Wang, Enterprise culture, Beijing university press, Beijing, 2012

[14] Y. CH. Yu, Introduction to e-commerce, China railway press, Beijing, 2013

[15] M. M. ZH, Y. J. CH, International financial management, Higher education press, Beijing, 2008

[16] H. A. CH, Principles of management, East China University of science and technology press, shanghai, 2013

[17] ZH. M. B, Y. H, Tutorial financial management practice, Beijing university press, Beijing, 2013

[18] J. M. W, Managerial economics, Beijing university press, Beijing, 2012

[19] B. T. H, Management of operational research, Higher education press, Beijing, 2010

[20] G. H. CH, J. L. B, Quality management, China agricultural university press, 2010

[21] H. CH, network marketing, Beijing university press, Beijing, 2013

[22] H. ZH. Y, Public relations theory and practice, Beijing university press, Beijing, 2011

[23] S. J. Y, fundamentals of accounting, Huazhong university of science and technology press, 2011

[24] T. R. Y, management accounting, Wuhan university press, Wuhan, 2012

[25] B. Wu, S. S. Wang, Purchasing and supply management, Zhejiang university press, Hangzhou, 2010

[26] C. L. R, Probability and mathematical statistics teaching methods discussed in this paper of financial and economics university, Cultural education, 2011 (18) : 171-173 